where $a, b$ are constants. This is an equation of the form (1), with a positive $\omega^{2}(t)$ of period $\pi$ and of mean-value $a$, if $0<b<a$. Since (6) reduces to $m=a-b$ and $M=$ $a+b$ in this case, it is seen from (5) that

$$
1-\vartheta^{2}=4 c /(1+c)^{2}, \text { where } c=b / a \text {. }
$$

Hence, condition (9) becomes $a<Q c^{1 / 2} /(1+c)$.

Since $c=b / a$, the latter inequality can be written in the form

$$
a / b<4 Q^{2} /(a+b)^{2} \text {, where } 0<b<a \text {. }
$$

Consequently, Mathieu's equation (19) is stable (and, what is more, "disconjugate") whenever its parameters $a, b$ satisfy the conditions (20), where $Q$ is the value (10), defined by (16) and (17).

\title{
REFERENCES
}

1. See E. R. Forsyth, Theory of differential equations, vol. IV, part III, pp. 425-431, 1902

2. E. R. van Kampen and A. Wintner, Am. J. Math. 59, 270-274 (1937)

3. See J. L. Geronimus, A. M. Ljapunow, Berlin, p. 80, 1954

4. G. Borg, Arkiv för Mat., Astr. och Fys. 31A, no. 1, 29 (1944)

5. See G. P6lya and G. Szegö, Aufgaben und Lehrsatze aus der Analysis, chap. II, no. 93, 1925

6. See C. F. Gauss, Werke, vol. XI , p. 145, pp. 542-543

\section{A HEAT CONDUCTION PROBLEM INVOLVING A SPECIFIED MOVING BOUNDARY*}

\author{
By R. E. GIBSON (Imperial College, London)
}

1. Introduction. Physical problems governed by an equation of heat conduction type occasionally arise which require a condition to be satisfied on a boundary moving at a specified rate. In the problems with which we are concerned the movement of the boundary is generated by the accretion there of new material and not by deformation of the medium. Typical examples are: the dissipation of excess pore water pressure in a clay layer upon which fresh material is being deposited [1], and the type of thermal problem considered by Benfield [2] relating to the theory of the formation of the earth on the dust cloud hypothesis. The moving boundary is also a feature of thermal problems where a change in state of the conducting medium occurs [3]. Here, the motion of the boundary separating the phases is not given and must be determined as part of the solution. We can hope, therefore, that our problems, where the motion of the boundary is given, will prove to be less intransigent.

While working on one-dimensional problems of this type, we found that exact solutions could be obtained in two cases, namely, when the rate of movement of the boundary is proportional to $t^{-1 / 2}$ and when it is constant. Although in few actual problems may these conditions be realized, we believe our results to be of interest since it appears unlikely that closed solutions can be obtained for an arbitrary motion of the boundary.

2. Problem with spherical symmetry. We consider first a case with spherical

*Received October 22, 1957. 
symmetry. For $t<0$ in $0 \leq r<\infty$ the temperature is constant, $v(r, t)=v_{0}$. At $t=0$ the formation of a sphere begins, within which an homogeneous rate of heat production $A(t)$ per unit volume occurs. Additional material, at a temperature $v_{0}$, arriving on the surface of the sphere also participates in the generation of heat at a rate $A(t)$, the current radius of the sphere being $R(t)$.

The appropriate equation for the temperature is

$$
\frac{1}{\kappa} \frac{\partial v}{\partial t}=\frac{1}{r^{2}} \frac{\partial}{\partial r}\left(r^{2} \frac{\partial v}{\partial r}\right)+\frac{A(t)}{K}, \quad 0 \leq r \leq R(t),
$$

where the constants $\alpha$ and $K$ are respectively the diffusivity and thermal conductivity of the medium. The problem is to determine $v(r, t)$ for $t>0$ subject to the conditions

$$
\begin{gathered}
v[R(t), t]=v_{0}, \\
\frac{\partial v}{\partial r}[0, t]=0 .
\end{gathered}
$$

3. Separation of variables. If in (1) we take as new variables $u=r\left(v-v_{0}\right), \xi=$ $r / R(t)$ we obtain

$$
\kappa \frac{\partial^{2} u}{\partial \xi^{2}}+\frac{\kappa A}{K} \xi R^{3}=R^{2} \frac{\partial u}{\partial t}-\xi R R^{\prime} \frac{\partial u}{\partial \xi},
$$

where the prime denotes differentiation with regard to $t$. The boundary of the sphere is now "fixed" at $\xi=1$. We now investigate the conditions for which

$$
u=f(t) G(\xi)+\xi h(t)
$$

is a solution of (4). This requires, firstly, that

$$
\frac{\kappa}{K} A(t)=R^{-2}\left(h^{\prime} R-h R^{\prime}\right) .
$$

The variables $f$ and $G$ are found to be separable only if $R=c t^{1 / 2}$, and then

$$
\frac{1}{G}\left[\frac{\kappa}{c^{2}} \frac{d^{2} G}{d \xi^{2}}+\frac{\xi}{2} \frac{d G}{d \xi}\right]=t f^{\prime} / f=\lambda,
$$

where $\lambda$ is an arbitrary constant. From the second of (8)

$$
f=D t^{\lambda},
$$

where $D$ is an arbitrary constant. Now, in terms of the new variables the boundary conditions (2) and (3) are

$$
\begin{gathered}
f(t) G(1)+h(t)=0, \\
f(t) G(0)=0 .
\end{gathered}
$$

From (9) and (11) we have

$$
h(t)=-D G(1) t^{\lambda}
$$

and therefore, from (5)

$$
u=D t^{\lambda}[G(\xi)-\xi G(1)] .
$$


The constant $I$ ) is found from (6) to be given by

$$
\frac{\kappa C}{K} A(t)=-D G(1) t^{\lambda-3 / 2}\left(\lambda-\frac{1}{2}\right) .
$$

The rate of heat production is therefore restricted to the form

$$
A(t)=A_{0} t^{\prime}, \quad 8>-1 .
$$

From (12)-(14) we then have

$$
u=\frac{\kappa A_{0}}{K} \frac{c t^{3 / 2+\cdot}}{(s+1)}\left[\xi-\frac{G(\xi)}{G(1)}\right] .
$$

The function $G$ is now found from (8), one of the arbitrary constants arising being determined from (11). Finally, we arrive at the following expression for the temperature,

$$
v=v_{0}+\frac{\kappa A_{0}}{K} \frac{t^{n+1}}{(s+1)}\left[1-\frac{{ }_{1} F_{1}\left(s+\frac{5}{2}, \frac{3}{2}, \frac{r^{2}}{4 \kappa t}\right)}{{ }_{1} F_{1}\left(s+\frac{5}{2}, \frac{3}{2}, \frac{c^{2}}{4 \kappa}\right)} \exp -\frac{1}{4}\left(\frac{r^{2}}{\kappa t}-\frac{c^{2}}{\kappa}\right)\right],
$$

which expression may be reduced to a finite combination of elementary functions if either 8 or $\left(s+\frac{1}{2}\right)$ is an integer [4]. In particular, for a constant rate of heat generation $(s=0)$

$$
v=v_{0}+\frac{\kappa A_{0}}{K}\left(\frac{c^{2} t-r^{2}}{c^{2}+6 \kappa}\right) .
$$

4. Integral equation. In the following section we derive a solution to (1), valid for an arbitrary rate of heat generation, when the motion of the boundary is

$$
R(t)=\mu t .
$$

We take

$$
\kappa A(t)=K F^{\prime \prime}(t)
$$

and consider a solution of (1) of the type

$$
\begin{aligned}
v=v_{0} & +F(t) \\
& -r^{-1} t^{-1 / 2} \int_{0}^{\infty} \rho^{-1} g(\rho)\left[\exp -(r-\rho)^{2} / 4 \kappa t-\exp -(r+\rho)^{2} / 4 \kappa t\right] d \rho .
\end{aligned}
$$

Apart from a constant factor the third term represents the temperature at $(r, t)$ due to an initial temperature $g(r)$ in the region $r \geq 0$ when heat generation is zero everywhere. Since the sphere vanishes at $t=0$ no initial condition on the temperature need be satisfied, and we are therefore at liberty to regard $g$ as an arbitrary function which must be chosen so that

$$
v[R(t), t]=v_{0}, \quad t>0
$$

and this requirement is met with if $g$ satisfies

$$
\frac{1}{2} R(t) F(t) t^{1 / 2} \exp \frac{R^{2}}{4 \kappa t}=\int_{0}^{\infty} \frac{g(\rho)}{\rho} \sinh \frac{\rho R}{2 \kappa t} \exp -\frac{\rho^{2}}{4 \kappa t} d \rho .
$$


In the special case* when $R=\mu t$ that part of the kernel involving the hyperbolic function may be absorbed in the unknown function. The following changes of variable

$$
\rho^{2}=\zeta, \quad t=1 / 4 \kappa p
$$

then reduce (18) to the form

$$
\int_{0}^{\infty} H(\zeta) e^{-\nu \zeta} d \zeta=\frac{\mu}{16 \kappa^{3 / 2} p^{3 / 2}} F\left(\frac{1}{4 \kappa p}\right) \exp \frac{\mu^{2}}{16 \kappa^{2} p},
$$

where

$$
H(\zeta)=\frac{g\left(\zeta^{1 / 2}\right)}{2 \zeta} \sinh \frac{\mu \zeta^{1 / 2}}{2 \kappa}
$$

Proceeding formally from (19) we find that

$$
g(\rho)=\frac{\mu \rho^{2}}{8 \kappa^{3 / 2}} \operatorname{cosech} \frac{\mu \rho}{2 \kappa} \cdot \frac{1}{2 \pi i} \int_{B r} p^{-3 / 2} F\left(\frac{1}{4 \kappa p}\right) \exp \left(\frac{\mu^{2}}{16 \kappa^{2} p}+\rho^{2} p\right) d p
$$

which, together with (17), leads to an expression for the temperature satisfying the governing equation (1) and the boundary conditions (2) and (3). In particular, when heat generation takes place at a constant rate

$$
F=A_{0} t / K
$$

the temperature is given by

$$
\begin{aligned}
& v=v_{0}+\frac{A_{0}}{K}\left[t-\frac{2}{\mu^{2} r}\left(\frac{\kappa}{\pi t}\right)^{1 / 2}\right. \exp -\frac{r^{2}}{4 \kappa t} \\
&\left.\cdot \int_{0}^{\infty} \rho \sinh \frac{\rho r}{2 \kappa t}\left\{\frac{\mu \rho}{2 \kappa} \operatorname{coth} \frac{\mu \rho}{2 \kappa}-1\right\} \exp -\frac{\rho^{2}}{4 \kappa t} d \rho\right] .
\end{aligned}
$$

5. One dimensional problems. Problems one-dimensional in some other coordinate system may be treated along lines similar to those discussed in the preceding sections.

For example, the temperature within an infinite cylinder of radius $R=c t^{1 / 2}$, when heat is generated according to (14), is found to be

$$
v=v_{0}+\frac{\kappa A_{0}}{K(s+1)} t^{s+1}\left[1-\frac{{ }_{1} F_{1}\left(s+2,1, r^{2} / 4 \kappa t\right)}{{ }_{1} F_{1}\left(s+2,1, c^{2} / 4 \kappa\right)} \exp -\frac{1}{4}\left(\frac{r^{2}}{\kappa t}-\frac{c^{2}}{\kappa}\right)\right]
$$

while for the conditions considered in Sec. 4 the temperature is given by

$$
\begin{aligned}
v=v_{0}+ & F(t) \\
& -\frac{1}{4 \pi i \kappa t} \exp -\frac{r^{2}}{4 \kappa t} \int_{0}^{\infty} \int_{B r} \frac{\rho}{p} F\left(\frac{1}{4 \kappa p}\right) \frac{I_{0}\left(\frac{r \rho}{2 \kappa t}\right)}{I_{0}\left(\frac{\mu \rho}{2 \kappa}\right)} \exp \left(\frac{\mu^{2}}{16 \kappa^{2} p}+p \rho^{2}-\frac{\rho^{2}}{4 \kappa t}\right) d \rho d p,
\end{aligned}
$$

wherein

$$
F(t)=\frac{\kappa}{K} \int_{0}^{t} A(\tau) d \tau .
$$

*The solution of Sec. 3 can also be attained directly from (18) after suitable changes of variable and the use of the convolution theorem. 
6. Numerical evaluation. In the spherical problem the quantities of physical importance are the temperature at the centre and the gradient at the boundary. When heat is generated at a constant rate and the radius increases according to (7), these quantities are found directly from (16). We note, firstly, that the temperature difference $\left[v(0, t)-v_{0}\right]$ is a constant fraction $1 /\left(1+6 \kappa c^{-2}\right)$ of the same difference if no leakage of heat across the boundary is allowed, and, secondly, as time goes on the temperature gradient at the boundary increases without limit.

On the other hand when $R=\mu t$ this gradient approaches an asymptotic value $-{ }_{\kappa} A_{0} / \mu K$ which may be seen by considering the behaviour of

$$
\frac{\mu K}{{ }_{\kappa} A_{0}} \frac{\partial v}{\partial r}[R, t]=\frac{2}{T}-1-\frac{8}{\pi^{1 / 2} T^{1 / 2}} e^{-\tau / 4} \int_{0}^{\infty} y^{3} e^{-\nu^{*}} \operatorname{cosech} y T^{1 / 2} \cdot d y
$$

as

$$
T\left(=\mu^{2} t / \kappa\right) \rightarrow \infty .
$$

The infinite integral has been evaluated numerically along the lines suggested by Goodwin [5], and the relation between the gradient and the time factor $T$ is shown in Fig. 1.

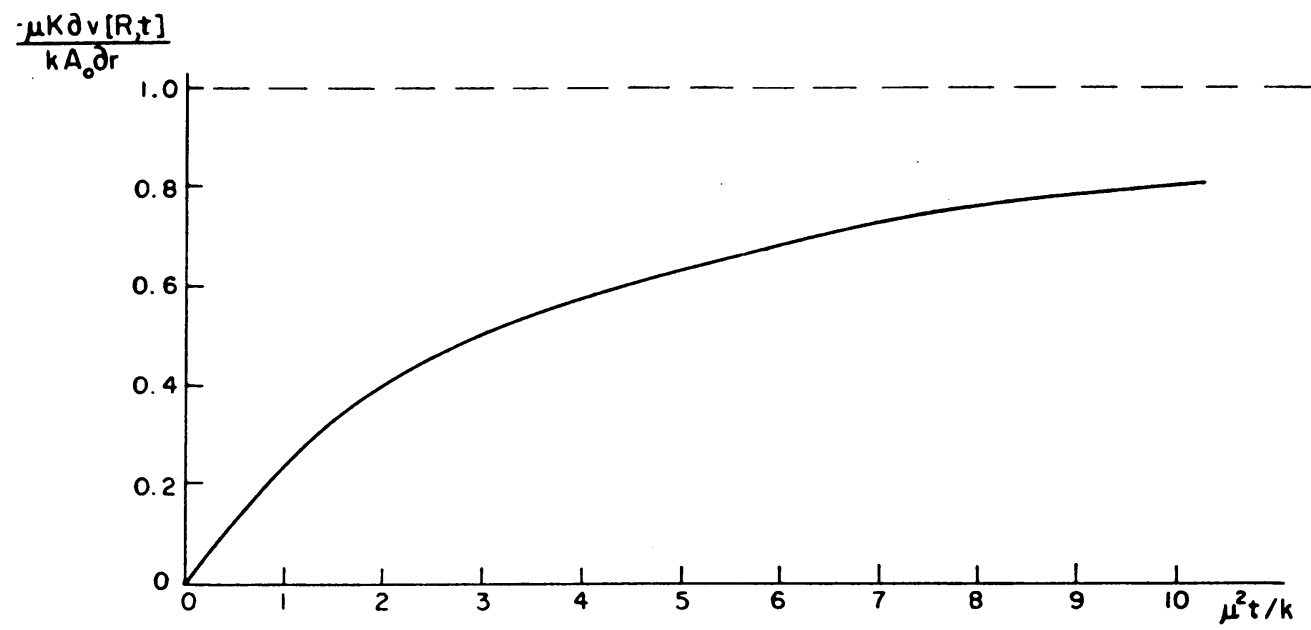

Fig. 1. Variation of boundary temperature gradient with time. The dashed line represents the asymptotic value.

\section{Bibliography}

1. K. Terzaghi, Erdbaumechanik auf boden physikalischer Grundlage, Franz Deuticke, Leipzig, p. 175 1924

2. A. E. Benfield, The temperature in an accreting medium with heat generation, Quart. Appl. Math. 7, 436-439 (1950)

3. See, for example, I. Kolodner, Free boundary problem for the heat equation with application to problems of change of phase, Communs. Pure and Appl. Math. 9, 1-31 (1956)

4. A. Erdélyi, Monatsh. Math. Phys. 46, 1-9 (1937)

5. E. T. Goodwin, The evaluation of integrals of the form $\int_{-\infty}^{\infty} f(x) e^{-x^{\prime}} d x$, Proc. Cambridge Phil. Soc. 45, 241 (1949) 